\title{
DETERMINING THE RESULTING DEFORMATION OF A RECTANGULAR METAL SHEET USING FEM CALCULATIONS
}

Ing. Ján Slamka, PhD. ${ }^{1}$, Prof. Ing. Marián Tolnay, CSc. ${ }^{2}$ Ing. Michal Bachratý, CSc. ${ }^{2}$, Assoc.Prof. Roland Jančo, PhD. ${ }^{1}$, prof. Pavol Kováč,PhD, MEng. ${ }^{2}$

${ }^{1}$ STU SjF ÚSETM, Nám. slobody 17, 81231 Bratislava, Slovak Republik

${ }^{2}$ FTS UNS DPE Trg Dositeja Obradovica 6, 21000 Novi Sad, Serbia

jan.slamka@sgteam.eu,marian.tolnay@stuba.sk,michal.bachraty@stuba.sk, roland.janco@stuba.sk,kovacp@uns.ac.rs

Keywords: finite element method, deformation, yielding, MATLAB, manipulation

\begin{abstract}
This article deals with creating a mathematical model, which is used for calculating the resulting maximum deformation of a rectangular metal sheet with the dimensions $1000 \times 900 \mathrm{~mm}$ and a thickness of $0.67 \mathrm{~mm}$, using FEM. The manipulated object is fixed using vacuum grasping elements, which will be also considered in this calculation. To reach these results we use the programs ANSYS and MATLAB.
\end{abstract}

\section{INTRODUCTION}

Problems encountered while finding the resulting deformation of a metal sheet are clearly mathematical, where complicated mathematical tasks are being performed. If we wanted to do these calculations manually, without the use of calculation and simulation programs, it would cost not only time but also there would be a wide presence of errors, where each calculation error is transferred to the next, thus rendering the whole simulation inaccurate. That is why, as a first step, a correct kinematic model of the sheet was defined.

A metal sheet with the dimensions $1000 \times 900 \times 0.67 \mathrm{~mm}$ was modeled In the program ANSYS. The output from the program was a macro in which the kinematic model computation element of the sheet, coordinates of the suction elements and mathematical mesh were defined. After entering the precise coordinates. ANSYS then created three files, which were read by MATLAB, since it is not possible to enter the FEM parameters directly, for example: the computation element.

\section{KINEMATIC EQUATIONS OF KIRCHHOFF'S AND VON KARMAN'S SHEET MODEL}

Both models are constructed in accordance to Kirchhoff's assumptions, which states that the orthogonal to the unloaded medial plane of the sheet (transverse normal) remain straight after being loaded, and is rotated in such a manner as to preserve the normal direction even on the deformed medial area (deformation area). It's also assumed that the points of the sheet lying on the same normal, do not change their relative positions (deformation over the thickness of the sheet is ignored $\varepsilon_{0}=0$ ). Then the field of displacement of the points can be expressed using the function of components of the displacement in the medial plane $\mathrm{u}_{0}, \mathrm{v}_{0}$ and $\mathrm{w}_{0}$. 


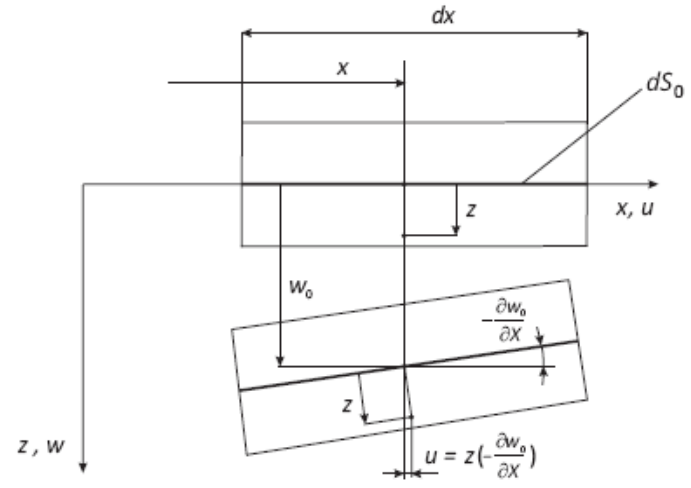

Figure 1: Expressed bending addition to displacement in $\mathrm{x}$ axis.

$$
\begin{aligned}
& w(x, y, z)=w_{0}(x, y)-z \frac{\partial w_{0}}{\partial x} \\
& v(x, y, z)=v_{0}\left(x_{t}, y\right)=z \frac{\partial w_{0}}{\partial x} \\
& u(x, y, z)=w_{0}(x, y)
\end{aligned}
$$

With this approximation function for the displacement, the Green-Lagrange tensor of deformation of the sheet is simplified.

$$
\begin{gathered}
E_{x}=\frac{\partial u}{\partial x}+\frac{1}{2}\left[\left(\frac{\partial u}{\partial x}\right)^{2}+\left(\frac{\partial v}{\partial x}\right)^{2}+\left(\frac{\partial w}{\partial x}\right)^{2}\right] \\
E_{y}=\frac{\partial v}{\partial y}+\frac{1}{2}\left[\left(\frac{\partial w}{\partial y}\right)^{2}+\left(\frac{\partial v}{\partial y}\right)^{2}+\left(\frac{\partial w}{\partial y}\right)^{2}\right] \\
E_{z}=\frac{\partial w}{\partial z}+\frac{1}{2}\left[\left(\frac{\partial u}{\partial z}\right)^{2}+\left(\frac{\partial v}{\partial z}\right)^{2}+\left(\frac{\partial w}{\partial z}\right)^{2}\right] \\
2 E_{x y}=\gamma_{x y}=\frac{\partial v}{\partial x}+\frac{\partial u}{\partial y}+\frac{\partial u}{\partial x} \frac{\partial u}{\partial y}+\frac{\partial v}{\partial x} \frac{\partial v}{\partial y}+\frac{\partial w}{\partial x} \frac{\partial w}{\partial y} \\
2 E_{y z}=\gamma_{y z}=\frac{\partial v}{\partial z}+\frac{\partial w}{\partial y}+\frac{\partial u}{\partial y} \frac{\partial u}{\partial z}+\frac{\partial v}{\partial y} \frac{\partial v}{\partial z}+\frac{\partial w}{\partial y} \frac{\partial w}{\partial z} \\
2 E_{x z}=\gamma_{x z}=\frac{\partial u}{\partial z}+\frac{\partial w}{\partial x}+\frac{\partial u}{\partial x} \frac{\partial u}{\partial z}+\frac{\partial v}{\partial x} \frac{\partial v}{\partial z}+\frac{\partial w}{\partial x} \frac{\partial w}{\partial z}
\end{gathered}
$$

(2)

In a geometrically and physically linear Kirchhoff sheet, the gradients of the function of displacement components are very small numbers, and all quadratic components are to be ignored (2).Using (1) we get

$$
\begin{gathered}
s_{x}=\frac{\partial u}{\partial x}=\frac{\partial u_{0}}{\partial x}-z \frac{\partial^{2} W_{0}}{\partial x^{2}} \\
s_{y}=\frac{\partial v}{\partial y}=\frac{\partial v_{0}}{\partial y}-z \frac{\partial^{2} W_{0}}{\partial y^{2}}
\end{gathered}
$$




$$
\begin{gathered}
\varepsilon_{z}=\frac{\partial w}{\partial z}=0 \\
\gamma_{x y}=2 s_{x y}=\frac{\partial v}{\partial x}+\frac{\partial u}{\partial y}=\frac{\partial v_{0}}{\partial x}+\frac{\partial u_{0}}{\partial y}-2 z \frac{\partial^{2} w_{0}}{\partial x \partial y} \\
\gamma_{x z}=2 \varepsilon_{y z}=\frac{\partial v}{\partial z}+\frac{\partial w}{\partial y}=-\frac{\partial w_{0}}{\partial y}+\frac{\partial w_{0}}{\partial y}=0 \\
\gamma_{x z}=2 \varepsilon_{x z}=\frac{\partial w}{\partial z}+\frac{\partial w}{\partial x}=-\frac{\partial w_{0}}{\partial x}+\frac{\partial w_{0}}{\partial x}=0
\end{gathered}
$$

(3)

\section{KIRCHHOFF'S THEORY FOR A SHEET LOADED ONLY IN THE SHEAR DIRECTION}

If there is a load acting upon a sheet in a transversal manner, and the points of the sheet can freely move in the directions $\mathrm{x}$ and $\mathrm{y}$, then the relative Kirchhoff's model deformations according to (3) are

$$
\begin{aligned}
& \varepsilon_{x}=-z \frac{\partial^{2} W_{0}}{\partial x^{2}} \\
& \varepsilon_{y}=-z \frac{\partial^{2} W_{0}}{\partial y^{2}} \\
& \gamma_{x y}=-2 z \frac{\partial^{2} W_{0}}{\partial x \partial y}
\end{aligned}
$$

(4)

For a certain value of variable $\mathrm{z}(-\mathrm{h} / 2 \leq \mathrm{z} \leq \mathrm{h} / 2)$ they are the deformation components of plane tension (where $\mathrm{s} \varepsilon_{\mathrm{z}}=0$ ), which, as seen from (4), linearly vary along the thickness of the sheet. From physical (constitutive) equations valid for plane tension and isotropic material we get the tension in the sheet.

$$
\begin{aligned}
& \sigma_{x}=\frac{E}{1-\mu^{2}}\left(\varepsilon_{x}+\mu \varepsilon_{y}\right)=-\frac{E z}{1-\mu^{2}}\left(\frac{\partial^{2} W_{0}}{\partial x^{2}}+\mu \frac{\partial^{2} W_{0}}{\partial y^{2}}\right) \\
& \sigma_{y}=\frac{E}{1-\mu^{2}}\left(\tau_{y}+\xi \varepsilon_{x}\right)=-\frac{E z}{1-\mu^{2}}\left(\frac{\partial^{2} W_{0}}{\partial y^{2}}+\beta \frac{\partial^{2} W_{0}}{\partial x^{2}}\right) \\
& \tau_{x y}=G_{y x}=\frac{E}{2(1+\mu)} \gamma_{x y}=-\frac{E z}{1-\mu^{2}}(1-\mu) \frac{\partial^{2} W_{0}}{\partial x \partial y}
\end{aligned}
$$

Where $\mathrm{E}$ is the elastic module, $\mu$ Poison number, and $\mathrm{G}$ shear elastic module. The deformation area is also the neutral area of tension, on which the tensions are null, and change sign. The extreme values of tension are analogically located on the upper surface of the sheet. 


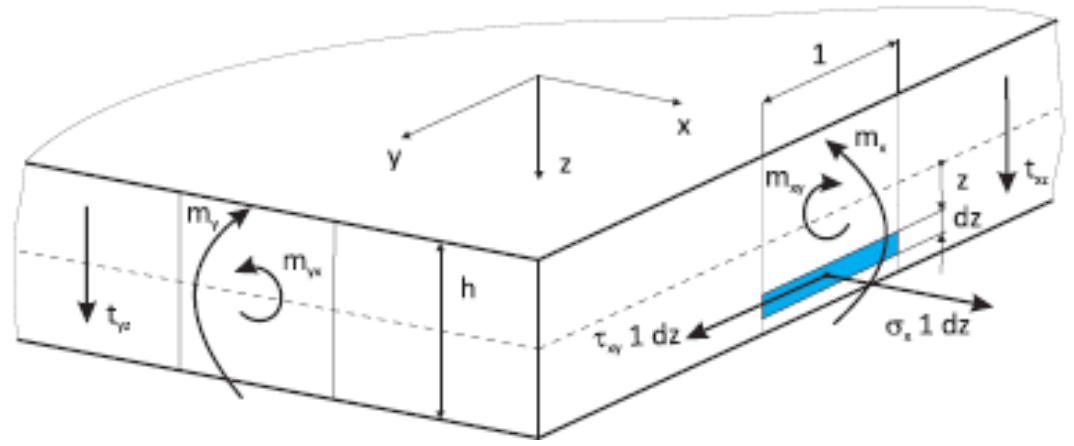

Figure 2: Resultant inner forces.

The resultant inner forces are defined by integrating shear tension with elementary width, (Fig. 2) and that is why they are usually labeled in small letters.

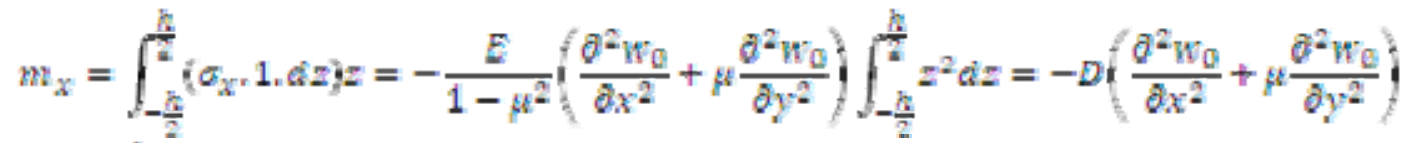

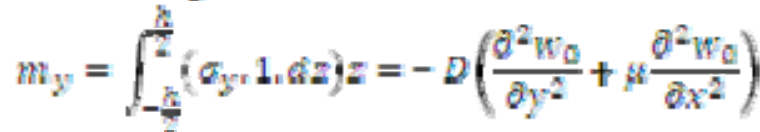

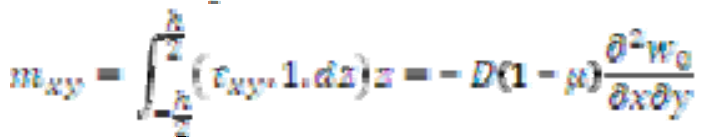

$$
\begin{aligned}
& t_{x z}=\int_{-\frac{\hbar}{2}}^{\tau_{x z}} 1 . d z=\frac{\partial m_{x}}{\partial x}+\frac{\partial m_{x y}}{\partial y}-D \frac{\partial}{\partial x}\left(\frac{\partial^{2} w_{0}}{\partial x^{2}}+\frac{\partial^{2} w_{0}}{\partial y^{2}}\right) \\
& E_{y z}=\int_{-\frac{\partial}{2}}^{\frac{\partial}{2}} \tau_{y z \cdot} \cdot d \cdot d z=\frac{\partial m_{y}}{\partial y}+\frac{\partial m_{x y}}{\partial x}=-D \frac{\partial}{\partial y}\left(\frac{\partial^{2} w_{0}}{\partial x^{2}}+\frac{\partial^{2} w_{0}}{\partial y^{2}}\right)
\end{aligned}
$$

(6)

Coefficient D, which in (6) expresses the stiffness of the sheet as

$$
D=\frac{E}{1-\mu^{2}} \int_{-\frac{k_{2}}{2}}^{\frac{\pi}{2}} z^{2} d z=\frac{E h^{2}}{12\left(1-\mu^{2}\right)}
$$

\section{DETERMINING THE ELEMENT MATRIX OF KIRCHHOFF'S SHEET, LOADED IN THE SHEAR DIRECTION USING FEM}

Matrices of the finite elements of Kirchhoff's sheet loaded in the shear direction are determined using the principal of virtual displacement.

Constitutive relation 


$$
\bar{\sigma}=\left\{\begin{array}{l}
m_{x} \\
m_{y} \\
m_{x y}
\end{array}\right\}=D\left[\begin{array}{ccc}
1 & \mu & 0 \\
\alpha & 1 & 0 \\
0 & 0 & \frac{1-\mu}{2}
\end{array}\right]\left\{\begin{array}{c}
\frac{-\partial^{\varepsilon} w_{0}}{\partial x^{2}} \\
\frac{-\partial^{2} w_{0}}{\partial y^{2}} \\
\frac{-2 \partial^{2} w_{0}}{\partial x \partial y}
\end{array}\right\}=D s
$$

On element the approximation of the function $\mathrm{w}_{0}(\mathrm{x}, \mathrm{y})$ is selected, using appropriate shape functions of element $\mathrm{N}(\mathrm{x}, \mathrm{y})$ and $\mathrm{n}$ values of generalized displacements of nodal points of the element.

$$
w_{0}(x, y)=\sum_{j=1}^{n} u_{2}^{n} N_{j}(x, y)
$$

$\mathrm{n}$ nodal equilibrium equation of the element is

$K^{*} u^{*}=f^{*}$

$\mathbf{K}^{\mathrm{e}}$ is the stiffness matrix of the element, $\boldsymbol{u}^{2}$ is the column matrix (vector) of generalized displacements of the element $f^{\text {f }}$ is the vector of nodal loads from the pressure p. For a general coefficient of the stiffness matrix $\mathrm{K}_{\mathrm{ij}}$ we got

$D_{11} \frac{\partial^{2} N_{i}}{\partial x^{2}} \frac{\partial^{2} N_{i}}{\partial x^{2}}+D_{22} \frac{\partial^{2} N_{i}}{\partial y^{2}} \frac{\partial^{2} N_{i}}{\partial y^{2}}+D_{12}\left(\frac{\partial^{2} N_{i}}{\partial x^{2}} \frac{\partial^{2} N_{i}}{\partial y^{2}}+\frac{\partial^{2} N_{i}}{\partial y^{2}} \frac{\partial^{2} N_{i}}{\partial x^{2}}\right)+4 D_{33} \frac{\partial^{2} N_{i}}{z}$

where $D_{i j}$ are the components of the material matrix of the constitutive $\mathbf{D}$.

Vector coefficient $\mathbf{f}^{\mathrm{e}}$ on $\mathrm{i}^{\text {th }}$ row is

$$
f_{i}=\int_{-g} p_{i} N_{i} d x d y
$$

Approximate polynom of deformation of the elementary medial area (9) can be written in matrix form

$$
\mathrm{w}_{0}(\mathrm{x}, y)=\mathrm{Nu}^{\mathrm{e}}
$$

where

$$
N=\left[N_{2}(x, y) N_{2} \ldots N_{n-1} N_{n}\right]
$$

is the row interpolation function of the element and

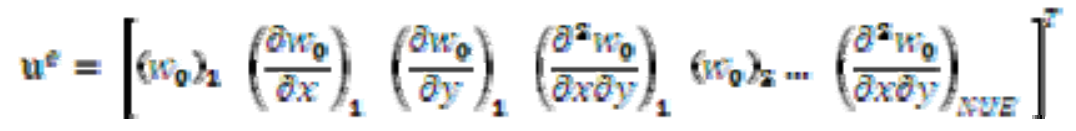

is the vector of generalized nodal displacement of the elements NUE nodal and with 4 degrees of freedom. These mathematic relations are used for FEM calculation in the program ANSYS.

5 USING THE PROGRAM ANSYS 
In this program the manipulated sheet was modeled to create a macro, in which it is possible to change the coordinates of the vacuum sucking elements and their diameter (Fig.3).

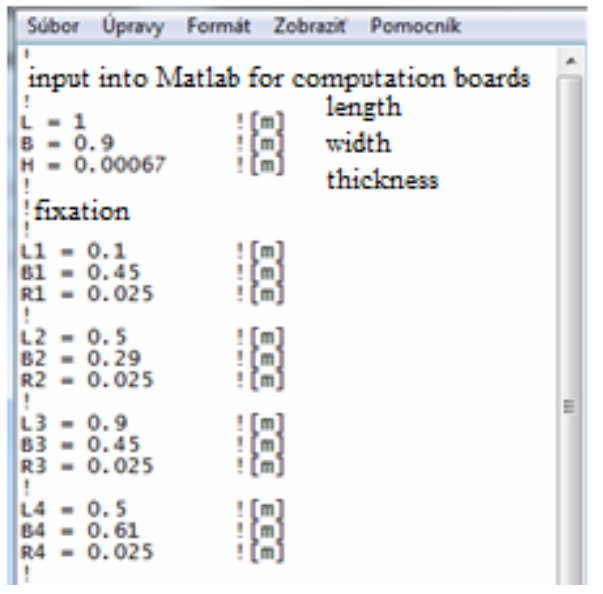

Figure 3: Coordinates of vacuum sucking elements and dimensions of the sheet.

After defining the input parameters of the program, three inputs to MATLAB were created. A file coordinates.dat was created, where the exact coordinates of the metal sheet and the sucking manipulators were defined. Then a file nodes.dat was created, in which the precise coordinates of nodal elements after mesh where given. In the last file the boundary condition were generated which are the conditions used for calculating the maximum resultant deformation.

\section{USING THE PROGRAM MATLAB}

The created program in MATLAB, directly calculated the resultant deformation using imported files from ANSYS.

On the metal sheet there were created several combinations of vacuum element placement, first they were moved in the diagonal directions of the metal sheet in six different places beginning at the end of the sheet, from the corners towards the middle until the elements touched. In the other four locations, the elements where moved along the axes of the sides with a $100 \mathrm{~mm}$ step. On Fig. 4 and 5 are examples of the location of the elements and the respective resultant deformation calculated in MATLAB.

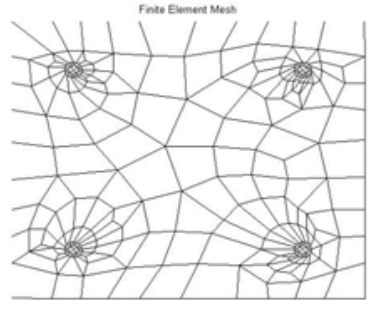

a)

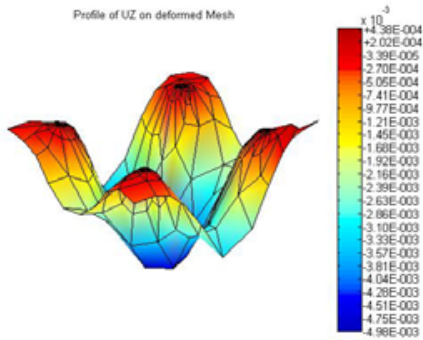

b)

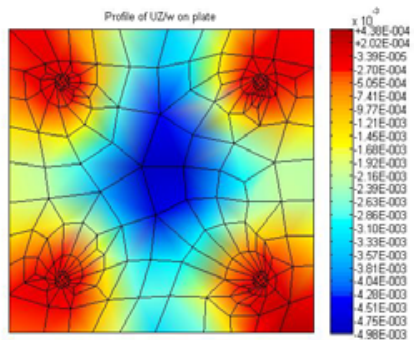

c)

In this case the resultant deformation is $4,98 \mathrm{~mm}$.

Figure 4: a) meshed sheet b) detail deformation c) resultant deformation 


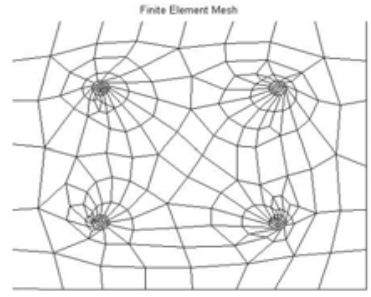

a)

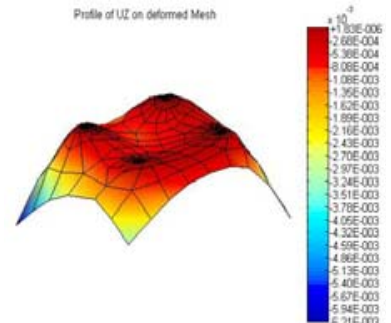

b)

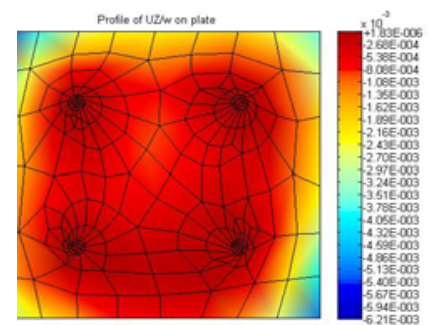

c)

In this case the resultant deformation is $6,21 \mathrm{~mm}$.

Figure 5: a) meshed sheet b) detail deformation c) resultant deformation

\section{CONCLUSION}

Using the results is possible when designing grasping vacuum suckers, and creating a newer computational model for the simulation of maximum deformation, so that experimental results compare well with real measurements. The appropriate thing to do would be to connect the program of the maximum deformation and the structural solution, resulting in an automatically resettable vacuum grasper that is able to recognize dimensions using a camera system and thus would be able to cope with different dimensions, and can automatically find the optimal position for manipulating the object.

\section{REFERENCES}

[1] Slamka, J.: Automatic manipulation of parts made from yielding material. Edícia kvalifikačných prác. SjF STU v Bratislave, 2012. ISBN 978-80 - 227- 3728-9.

[2] Reddy, J. N.: An Introduction to Nonlinear Finite Element Analysis. Oxford University Press, 2004. ISBN 0-07-246685-5.

[3] ANSYS, MATLAB : Theoretical Manual. 